\title{
Cambios históricos en el esfuerzo pesquero de la flota parguera de Taganga, Caribe Colombiano
}

\section{Historical changes in the fishing effort of the parguera fleet of Taganga, Colombian Caribbean}

\author{
Andrés F. Acevedo-Mendivelso; Félix Cuello; ;airo Altamar ${ }^{3}$
}

'Ing. Pesquero. Universidad del Magdalena. Santa Marta - Magdalena, Colombia; e-mail: felipe9441@gmail.com; @ https://orcid.org/0000-0002-8460-5562

${ }^{2}$ Ing. Pesquero, Ph.D.(c). Universidad del Magdalena, Laboratorio de Investigaciones Pesqueras Tropicales. Santa Marta - Magdalena, Colombia; e-mail: felcuello@gmail.com; (1) https://orcid.org/0000-0002-4364-6229

${ }^{3}$ Ing. Pesquero, Ph.D.(c). Universidad del Magdalena, Laboratorio de Investigaciones Pesqueras Tropicales. Santa Marta - Magdalena, Colombia; e-mail: jaltamar@unimagdalena.edu.co; D https://orcid.org/0000-0002-0886-2719

Cómo citar: Acevedo-Mendivelso, A.F.; Cuello, F.; Altamar, J. 2020. Cambios históricos en el esfuerzo pesquero de la flota parguera de Taganga, Caribe Colombiano. Rev. U.D.C.A Act. \& Div. Cient. 23(2):e1200. http://doi.org/10.31910/rudca.v23.n2.2020.1200

Artículo de acceso abierto publicado por Revista U.D.C.A Actualidad \& Divulgación Científica, bajo una licencia Creative Commons CC BY-NC 4.0

Publicación oficial de la Universidad de Ciencias Aplicadas y Ambientales U.D.C.A, Institución de Educación Superior Acreditada de Alta Calidad por el Ministerio de Educación Nacional.

Recibido: Abril 9 de 2019 Aceptado: Julio 18 de 2020 Editado por: Ingeborg Zenner de Polanía

\section{RESUMEN}

La pesquería artesanal avanzada de lanchas "pargueras" que opera en el área norte del mar Caribe de Colombia ha experimentado grandes dificultades relacionadas con el volumen de los desembarcos, que han llevado al colapso económico de la flota. La disminución de la abundancia de los recursos ícticos ha ocasionado la expansión del área de pesca, el aumento en la duración del viaje de pesca, la disminución de los ingresos y el incremento de los gastos de operación. Existen vacíos en el conocimiento de la distribución espacial actual e histórica del esfuerzo pesquero de la flota, que no permiten asociarlos a los cambios en las abundancias, la composición de las capturas y la disminución de tamaños de las especies capturadas. Mediante entrevistas a pescadores y con la ayuda del conocimiento ecológico tradicional, el conocimiento local y el uso de sistemas de información geográfica, se mapeó la distribución espacial del esfuerzo pesquero y su comportamiento desde los años
70 hasta la actualidad. Como principal resultado, se evidenció un aumento promedio de $134,5 \mathrm{~km}$ en la distancia del viaje de pesca en casi 50 años y cambios en el área y profundidades de pesca, que variaron desde los 27,8 a 1117,8 $\mathrm{km}^{2}$ y de los 93,8 a $41,1 \mathrm{~m}$, respectivamente. Los resultados obtenidos constituyen insumos valiosos para la implementación de estrategias de manejo, orientadas a asegurar la sostenibilidad ecológica y la viabilidad económica de esta importante pesquería.

Palabras clave: Pesca artesanal; Esfuerzo pesquero; Palangre; Conocimiento ecológico tradicional; Caribe colombiano.

\section{ABSTRACT}

The advanced artisanal fishery of fishing boats called "pargueras" that operates in the northern area of the Colombian Caribbean has experienced great difficulties related to the volume of landings that 
have led to the economic collapse of the fleet. The decrease in the abundance of fish resources has caused an expansion of the fishing area, increased duration of the fishing trip, decreased incomes and the increase in operating expenses. Existing knowledge gaps with respect to current and historical spatial distribution of the fleet's fishing effort prevent an association to changes in resource abundances, composition of catches and decreased species catch sizes. Through interviews with fishermen and based in traditional ecological knowledge, local knowledge and the use of geographic information systems, we mapped the spatial distribution of fishing effort and its inter-decadal behavior, from the 70s to present. The main result was a $134.5 \mathrm{~km}$ increase in the distance of an average fishing trip in almost 50 years and changes in the surface area and fishing depths which varied from 27.8 to $1117.8 \mathrm{~km}^{2}$ and 93.8 to $41.1 \mathrm{~m}$, respectively. The results obtained constitute valuable inputs for the implementation of management strategies aimed at ensuring the ecological sustainability and economic viability of this important fishery.

Keywords: Artisanal fishing; Fishing effort; Longline; Traditional ecological knowledge; Colombian Caribbean.

\section{INTRODUCCIÓN}

La pesca artesanal mundial contribuye a la seguridad alimentaria, a la erradicación de la pobreza y a los ingresos y empleo a millones de personas (FAO, 2018). Se estima, que $260 \pm 6$ millones de personas, se dedican a las pesquerías marinas globales, trabajando tiempo completo y parcial, ya sea directa o indirectamente, de los cuales, $22 \pm 0,45$ millones son pescadores artesanales o de pequeña escala (Teh \& Sumaila, 2013); no obstante, este sector enfrenta retos, como la disminución de los recursos pesqueros; la degradación de los hábitats acuáticos y la fragmentación de políticas sociales, (Suárez de Vivero, 2002), esto último, en términos de la falta de una política de Estado efectiva y de una débil estructura de gobernanza, que resulta ineficiente para administrar los recursos pesqueros y los usuarios asociados a las pesquerías (Saavedra et al. 2016). En Colombia, la pesca artesanal ha tenido una influencia importante en la economía y la cultura del país, llegando a ser considerada necesaria, tanto para la subsistencia y la seguridad alimentaria como para el desarrollo de una actividad comercial (FAO, 2006).

Las lanchas "pargueras" de Taganga, se dedican a la pesca de pargos, tales como el rayado (Lutjanus synagris), cebal o palmero (L. analis) y cunaro (Rhomboplites aurorubens), meros (Serranidae) y medregales (Seriola spp.). Estas embarcaciones operan todo el año y constituyen las unidades de pesca de mayor autonomía en los departamentos del Magdalena y La Guajira (Manjarrés, 1993), denominadas también, en otros estudios, como flota artesanal avanzada (Barros \& Manjarrés, 2004). La duración de las faenas de pesca oscila entre 7 y 15 días, dependiendo, entre otros factores, del tamaño de la embarcación, cuyas esloras varían entre 8,2 y 12,7m; del número de tripulantes a bordo que, según la acomodación, puede ser de 5 a 6 pescadores y del arqueo, que puede variar entre 1,8 a 7,2 Toneladas de Registro Neto (Arévalo et al. 2004). Esta flota captura especies de peces demersales, tales como pargos, meros y medregales, utilizando palangres horizontales de fondo y líneas de mano tipo "ballestilla" (Arévalo et al. 2004).

En la evaluación y el ordenamiento de los recursos pesqueros es importante contar con información temporal y espacial suficiente; por ejemplo, los modelos espaciotemporales proporcionan información sobre los patrones de distribución espacial del pargo rojo en el Golfo de México (Grüss et al. 2018). Muchos estudios han hecho énfasis en la importancia de considerar, además de los cambios temporales, los patrones espaciales de las poblaciones explotadas y sus indicadores (Babcock et al. 2005). En el caso de las pesquerías artesanales de Colombia, no se ha consolidado un sistema estadístico basado en el monitoreo continuo en el tiempo, que considere la distribución espacial del esfuerzo pesquero de las flotas, siendo que estas variables resultan determinantes para el ordenamiento de una flota. En el presente trabajo, se caracterizan los cambios espaciales históricos del esfuerzo pesquero de las lanchas pargueras que operan desde Taganga, así como su efecto sobre la duración del viaje de pesca. El análisis, se realizó desde la perspectiva del conocimiento tradicional del pescador artesanal sobre el entorno ecológico, donde, habitualmente, se realizan las capturas de peces demersales, en particular, los pargos de la familia Lutjanidae.

\section{MATERIALES Y MÉTODOS}

Área de estudio. En el escenario del Caribe colombiano, Taganga aporta cerca del 17\% del total de los desembarcos pesqueros artesanales, por lo cual, se constituye en una de las más importantes comunidades pesqueras de esta región (De la Hoz et al. 2015). Está ubicada al norte de la ciudad de Santa Marta y se encuentra enmarcada por los cerros Punta Venados (N11¹6'14" W74¹2'16”) y Punta Colorada (N11'15'44" W74'11'56"), que se derivan de las estribaciones de la Sierra Nevada de Santa Marta (Gaitán-Espitia, 2008). Las pargueras desarrollan su actividad en la zona costera del área norte del Caribe colombiano, con puerto base en la bahía de Taganga. Faenan en el área comprendida entre las poblaciones de Palomino y Manaure (Arévalo et al. 2004) y, ocasionalmente, en los sectores de Manaure y el cabo de La Vela (Figura 1).

En el área, se presentan dos centros de surgencia localizados, uno frente a la Península de La Guajira y, el otro, en cercanías del Parque Nacional Natural Tayrona (PNNT) (Sarmiento et al. 2013). El ancho de la plataforma continental varía de acuerdo con la región; es muy estrecha frente al PNNT y muy amplia frente a las costas de La Guajira; por esta razón, las operaciones de pesca de la flota parguera, se realizan en una extensa área, que va desde los 20 hasta los $200 \mathrm{~m}$ de profundidad (Arévalo et al. 2004).

Recolecta de información. Con el fin de rescatar el conocimiento ecológico tradicional (CET), se diseñó un formulario para entrevistas semiestructuradas, para permitir al pescador expresarse en las causas de la expansión del esfuerzo de pesca y se implementó un muestreo, tipo "bola de nieve", que permite la ubicación de otros informantes clave e información destacada, sobre la operación de la pesquería (LADDER, 2001), dirigido a pescadores artesanales de la flota parguera de Taganga. Las aproximaciones desde el CET 


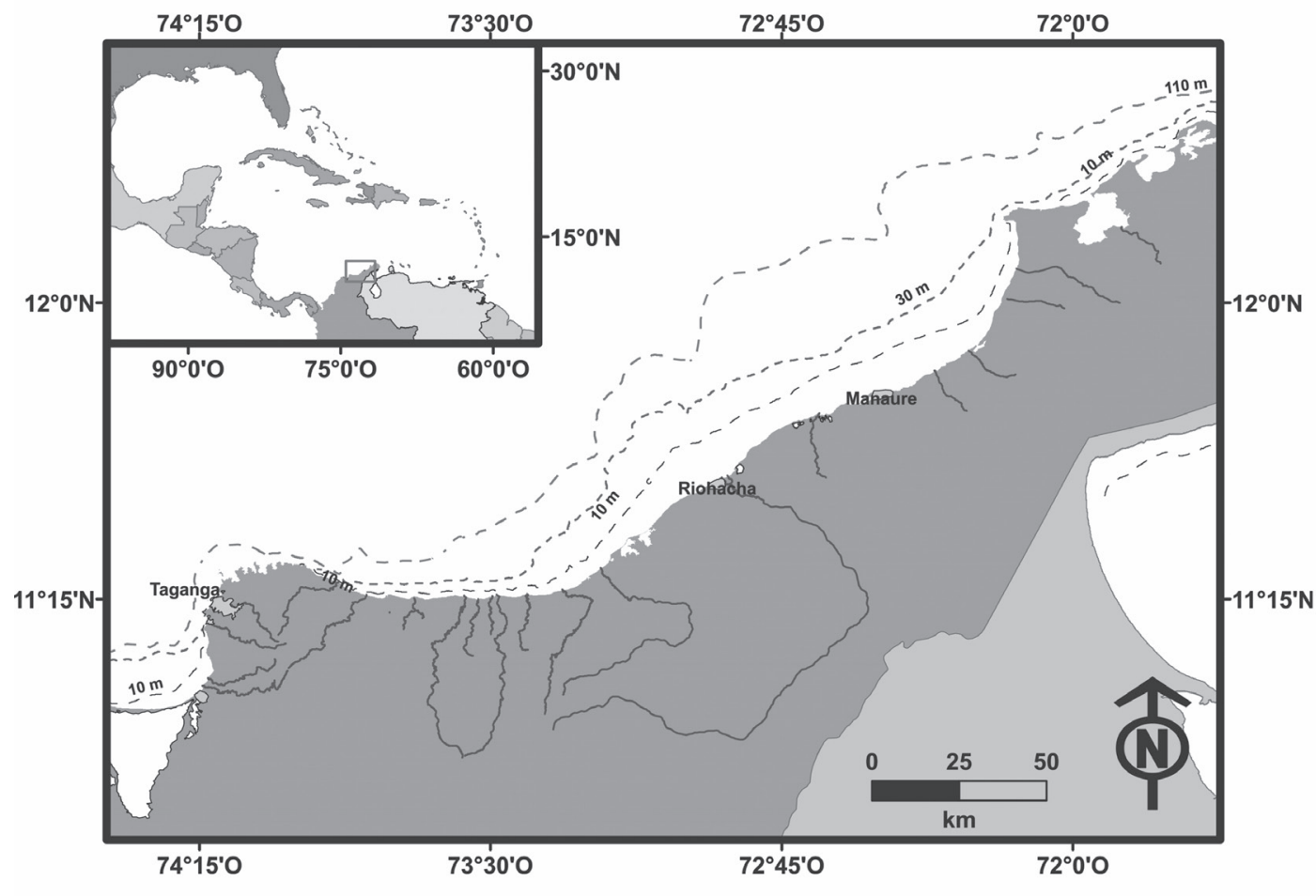

Figura 1. Área de estudio que muestra dónde se realizan las operaciones de pesca de la flota de lanchas pargueras, comprendidas entre Taganga y el cabo de La Vela.

reconocen la validez de la información empírica de las comunidades locales, cuyos medios de subsistencia dependen directamente de los recursos naturales (Teixeira et al. 2013; Ramires et al. 2015) que, además, son útiles para comprender los patrones espaciales y temporales de las pesquerías (Lima et al. 2017) o también, para conectar con modelos tróficos, que pretenden mejorar la gestión de los recursos pesqueros (Sánchez-Jiménez et al. 2019). Se indagaron los siguientes datos acerca de los pescadores y sus actividades: edad, tiempo de experiencia, características de la flota (año de inicio, procedencia y tamaño de las embarcaciones, nombre y número de embarcaciones activas e inactivas), artes de pesca utilizados, sitios de pesca, duración de los viajes, variación inter e intra-anual de las capturas, causas de la disminución de la abundancia y el tamaño de los recursos y de la inactividad de las embarcaciones de la flota.

Para conocer la expansión histórica de la frontera pesquera, se consultó a los pescadores por la información espacial de las capturas en cada década (desde 1979 hasta 2018); para ello, se utilizó un mapa con cuadrículas identificadas con códigos alfanuméricos, incluyendo georreferenciaciones costeras, batimétricas y las líneas de costa, a fin de que los entrevistados pudieran precisar mejor los sitios de pesca. Los datos utilizados en el presente estudio fueron obtenidos, previo consentimiento verbal de los pescadores y bajo estrictos términos de confidencialidad. Los registros fueron digitalizados y almacenados en una hoja de cálculo Microsoft Excel (Microsoft ${ }^{\circledR}$ Office Excel ${ }^{\circledR}$ 2007).
En el caso de la distribución espacial del esfuerzo, los datos fueron procesados mediante el software QGIS 2.18.15 (Quantum GIS Development Team, 2017), cuyo sistema de información geográfica (SIG) permitió obtener mapas decadales, que muestran los cambios en la distribución espacial del esfuerzo pesquero histórico de la flota. Asimismo, utilizando el SIG, se comparó el área y la profundidad media de las operaciones pesqueras, señaladas por los pescadores, desde 1970 hasta 2018, década de los 70 hasta la actual.

\section{RESULTADOS Y DISCUSIÓN}

En total, se entrevistaron 16 pescadores, que representan el 45\% de la flota, con una edad promedio de 58 años, de los cuales, el $25 \%$ se encuentran inactivos por la falta de embarcaciones activas donde trabajar. Se destaca, que los pescadores encuestados iniciaron sus labores desde muy jóvenes (19 $\pm 4,5$ años D.E.) y suelen ser personas con muchos años de experiencia en actividades relacionadas con la pesca y, en promedio, $35 \pm 12$ años D.E., pescando en pargueras. Los años de experiencia como pescador de lanchas pargueras, les permite precisar mejor cómo se originó esta flota en Colombia y, particularmente, en la bahía de Taganga.

En general, la población entrevistada tiene más de 35 años de experiencia asociada a la actividad pesquera, por lo cual, la valoración del CET de los pescadores de lanchas pargueras, en temas relacionados con la selección de los sitios históricos de pesca (distribución espacial del esfuerzo pesquero), resulta ser coherente con la infor- 
mación científica disponible. En consecuencia, es posible afirmar que el reconocimiento de la experiencia del pescador es fundamental para determinar aspectos a considerar, como la sostenibilidad de los recursos pesqueros y la situación social y económica del pescador (Hall \& Close, 2007).

El 50\% de los entrevistados identifica la llegada de las pargueras a Colombia a inicios de la década de los 70. El 100\% de los pescadores coincidieron en que las lanchas proceden de Venezuela. Algunos de ellos (81\%), específicamente, aseveraron que provenían de Punto Fijo (Estado Falcón, Venezuela) que, en principio, eran propiedad de venezolanos y que los capitanes de las lanchas eran de esa misma nacionalidad. Los pescadores mayores de 61 años señalan que las primeras lanchas pargueras fueron las nombradas "Alaska", "Clarivel", "Miosotis", "Luis Rafael” y "La Meris", tres de ellas, sin puente de mando en la cubierta. Aunque no existe documentación oficial, las afirmaciones de cuatro de los pescadores entrevistados señalaron que, inicialmente, la flota fundamentó sus operaciones pesqueras, debido a la comercialización de pargos y meros, con destino a Barranquilla.
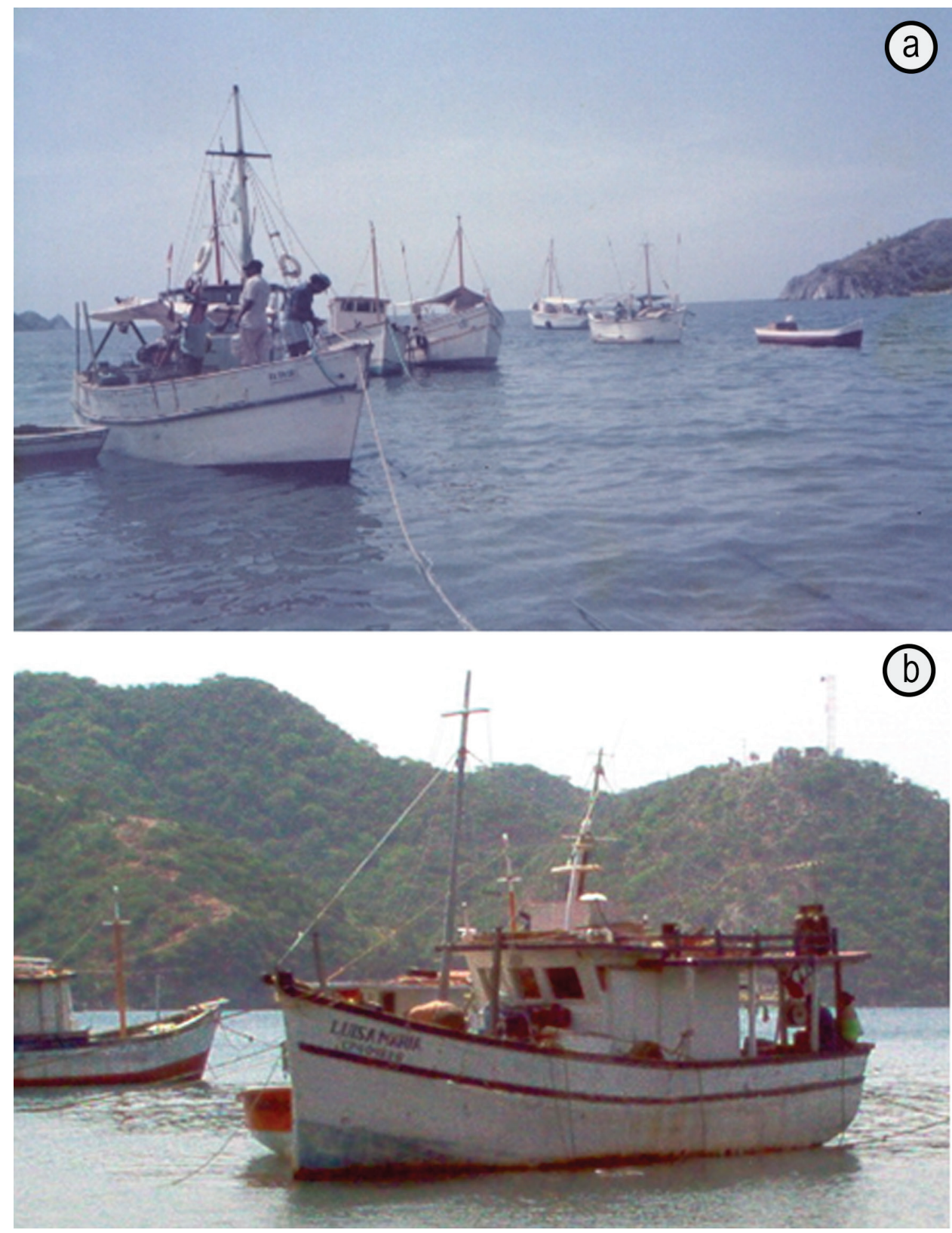

Figura 2. Embarcaciones típicas de la flota parguera. a) modelo histórico sin puente de mando; b) modelo con puente para mejor acomodación y seguridad de la tripulación. 
Las lanchas pargueras de Taganga son las unidades de pesca con mayor autonomía de la flota artesanal que opera en la costa marítima colombiana de los departamentos del Magdalena y La Guajira (Manjarrés, 1993; Gómez-Canchong et al. 2004). Estas embarcaciones utilizan motores internos con cascos de madera recubierta de fibra de vidrio, que pueden o no tener puente sobre la cubierta ( $60 \%$ con puente), lo que influye en la comodidad y la seguridad de la tripulación, cuando se realizan prolongadas jornadas de pesca, que pueden llegar a ser de hasta 2 semanas (Figura 2).

Al contrastar la información obtenida mediante las herramientas del CET y la información bibliográfica, se identificaron diferencias con relación al origen y la procedencia de esta flota. En ambos casos, se señala la década de los 70 , como el origen de las operaciones pesqueras, pero en el imaginario de los pescadores es Venezuela, de donde provinieron las primeras embarcaciones y las más nombradas son: "Alaska", "La Dalia", "Guadalquiri", "Llava", "Misiotti", "Sara Isabel", "El Topo", "Felicidad", "Argentina", "La Meris", "Pléyades" y "Claribel". Arévalo et al. (2004) señalan que el origen está asociado a la llegada de las embarcaciones "Alaska", "Meris", "Luis Rafael" y "Sara Isabel", procedentes de Aruba. El dato coincidente en ambas fuentes es que la embarcación "Alaska" fue la primera que inició operaciones desde Taganga.

El tamaño de la flota ha variado a través del tiempo y, hoy día, solamente dos de las primeras que llegaron a Taganga siguen en el inventario, pero solo una se encuentra activa; sin embargo, desde sus inicios en la década de los 70 hasta el 2020, se puede considerar que la década de los 80 es el periodo de máximas capturas y número de embarcaciones. El 75\% destaca que desembarcos por encima de los $2.000 \mathrm{~kg}$ eran frecuentes y el $37,5 \%$ coinciden en que
15 ha sido el número máximo de embarcaciones pargueras activas. Desde la década de los 70, las embarcaciones utilizaron el palangre como el arte de pesca principal, que consiste en una línea principal, de la cual, se derivan líneas secundarias o bajantes, en cuyos extremos, se anudan los anzuelos. El 85\% de los entrevistados afirmó tener preferencias hacia el palangre, por ser un arte de pesca más eficiente, en tanto que el $62,5 \%$ suelen combinar este arte con la línea de mano "ballestilla"; este arte corresponde a una línea de mano modificada en la sujeción del lastre, mediante el uso de una varilla de cobre o acero inoxidable, que libera de tensión las líneas secundarias, donde se anudan los anzuelos.

El máximo esplendor de esta flota, se remonta a la década de los 90, cuando los inventarios de la flota alcanzaron un total de 18 embarcaciones (1990), de las cuales, solamente tres se encontraban inactivas (Viloria, 1993). Campo (1996) censó 12 unidades en 1995 y el número de lanchas inactivas llegó a cuatro; a finales de la década (1999), Arévalo et al. (2004) reportaron las mismas 12 embarcaciones, pero las inactivas incrementaron a seis. El colapso de la pesquería proviene de la reducción de la renta económica, producto de la disminución de los desembarcos por faena de la flota, que se traduce en menores ingresos y el aumento de los gastos de operación (Zúñiga et al. 2013), circunstancias que han conllevado a la disminución del tamaño de la flota que, actualmente, solo tiene tres embarcaciones activas y dos inactivas (Figura 3).

Históricamente, el palangre y la ballestilla han sido los artes de pesca utilizados por esta flota (Gómez-Canchong et al. 2004); el $62,5 \%$ de los pescadores utilizan la combinación de estos artes. Según Arévalo et al. (2004), el uso de uno u otro arte depende de la profundidad de pesca; el palangre suele ser operado en aguas

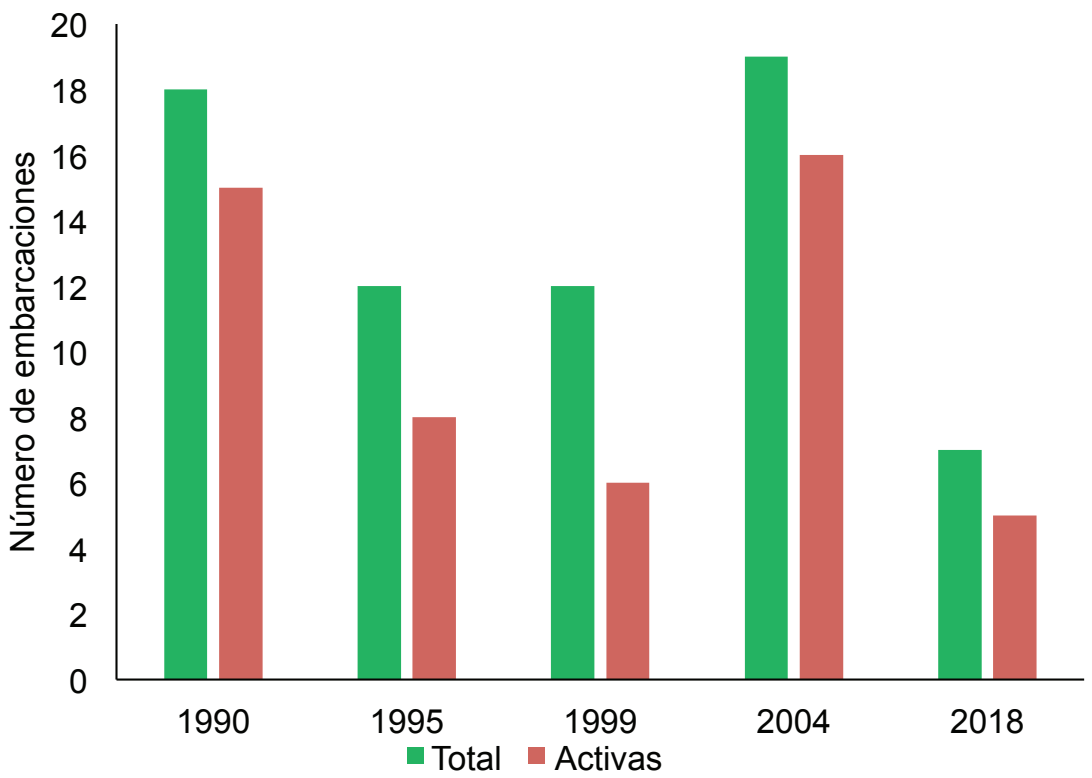

Figura 3. Variación histórica del tamaño de la flota parguera que opera desde la bahía de Taganga. 
someras (entre 27 y $60 \mathrm{~m}$ ), mientras que la ballestilla, se utiliza a profundidades mayores de $60 \mathrm{~m}$, particularmente, en épocas o zonas en que abundan los "sapos" (Lagocephalus laevigatus), especie íctica, que destruye los palangres, cortando el material de la línea madre y los bajantes (o reinales). Algunos pescadores entrevistados señalaron que el palangre es inoperable, cuando aumenta la fuerza de la corriente, lo cual, condiciona el uso exclusivo de la ballestilla.

La duración promedio del viaje de pesca en los inicios de las operaciones de las lanchas pargueras era de cuatro días, oscilando entre dos y seis días. Actualmente, el promedio de días fuera de puerto es de 13, de los cuales, dos se emplean para ir y volver de los sitios de pesca. Según la percepción de los pescadores ha ocurrido un cambio histórico en los sitios pesca (Figura 4). Al transcurrir el tiempo, las faenas se han ido expandiendo hacia el nororiente, pasando de operar, inicialmente, en el parque Tayrona a caladeros, que llegan hasta las inmediaciones del Cabo de La Vela (La Guajira).

La distribución espacial también mostró un aumento en el tamaño de las áreas de operación de la flota (Figura 5a), que varió desde 27,8 hasta $1117,8 \mathrm{~km}^{2}$, probablemente, por la necesidad de aumentar la exploración de nuevos fondos. Asimismo, se estimaron cambios en las profundidades de pesca, que variaron de 93,8 a 41,1m. En este caso, el promedio de la profundidad disminuyó, debido a que la expansión de la flota se realizó hacia un sector de La Guajira, donde la plataforma continental es amplia y somera.
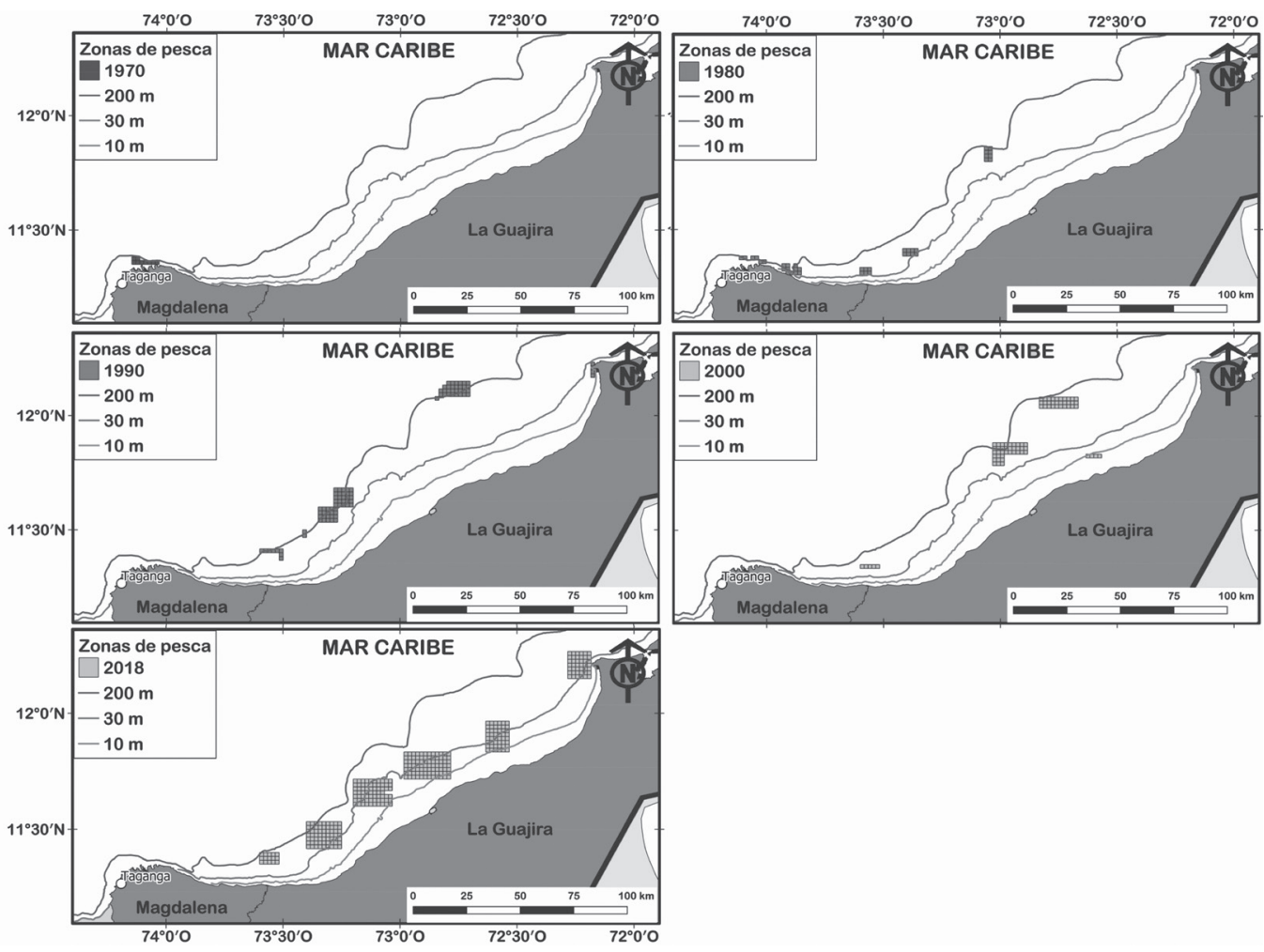

Figura 4. Mapas elaborados a partir de la percepción de los pescadores. Se evidencia la expansión espacial de los caladeros de la flota de lanchas pargueras de Taganga, desde 1970 hasta 2018.
Los resultados indicaron que ha ocurrido un aumento, tanto en la duración de las faenas como en la distancia del viaje de pesca. El incremento de la distancia, se estimó en $134,5 \mathrm{~km}$ en casi 50 años de operaciones de la flota (Figura 5b), lo que aumenta los gastos de operación, principalmente, el combustible.

En otros estudios, se ha señalado que la duración del viaje varía entre 7 y 15 días, lo cual, depende del tamaño y la autonomía de la embarcación (Arévalo et al. 2004) y de la comercialización de la pesca, que en otras décadas, se hacía en Aruba (Viloria, 1993); sin embargo, utilizando el SIG, a partir del conocimiento la percepción de los pescadores se ha determinado un aumento, tanto del área cubierta por la flota como de la distancia a los sitios de pesca.

De acuerdo con la percepción de los entrevistados, en casi tres décadas, los desembarcos de las lanchas pargueras han disminuido un 51\% (Figura 6); sin embargo, como ocurre habitualmente con las capturas, en las respuestas de los pescadores hay gran variabilidad. Estos declararon que, en 1990, los desembarcos fluctuaban entre 90 y $5000 \mathrm{~kg}$ por faena o viaje de pesca, mientras que en la actualidad, fluctúan entre 20 y $2000 \mathrm{~kg}$. Por otro lado, es evidente que existe una variación intra-anual en las abundancias de pargos ( $L u$ tjanus synagris y L. analis), meros (Serranidae) y medregales (Seriola spp.) que, según la percepción de los pescadores existen dos picos de captura, que alcanzan sus máximos en abril y octubre. 

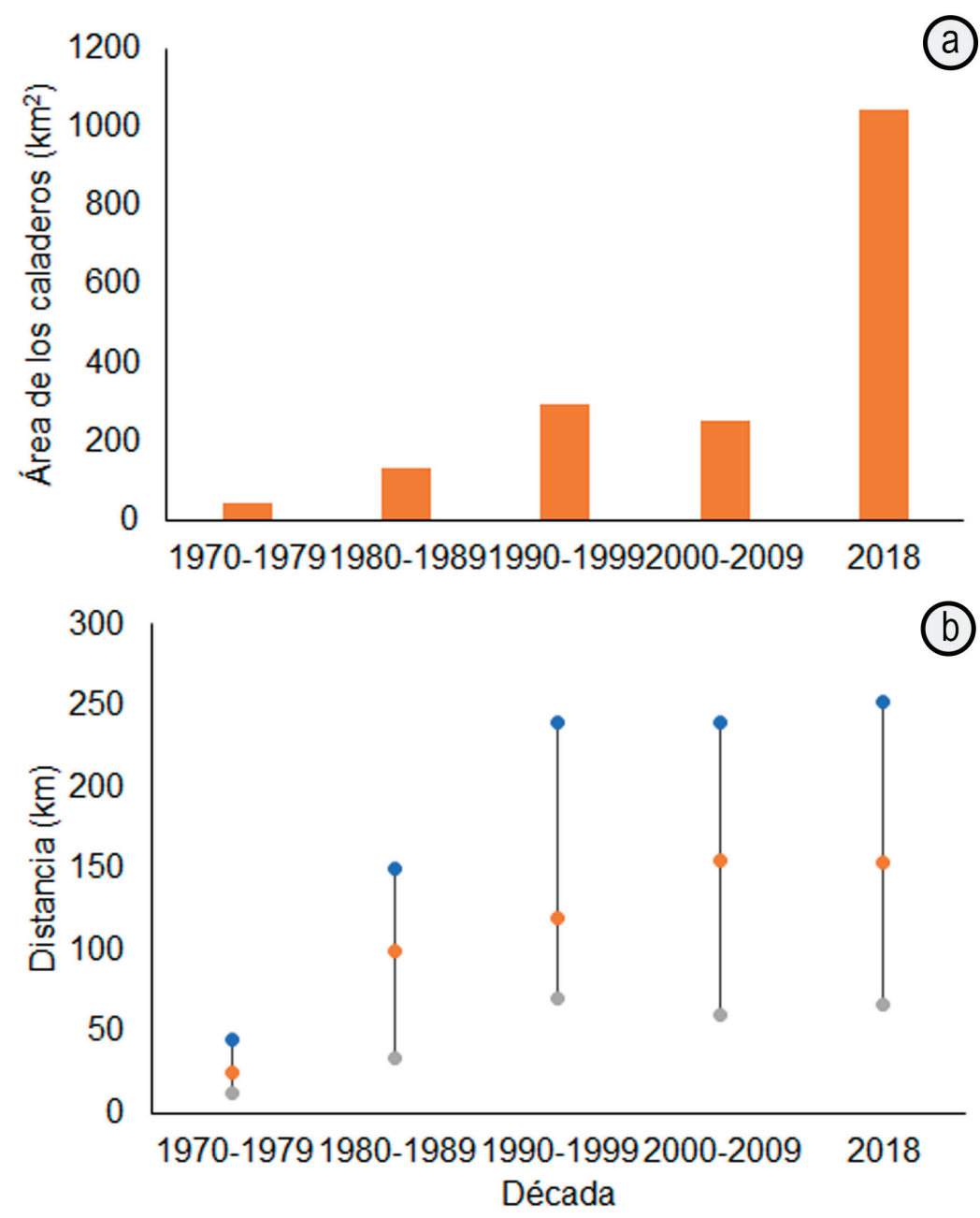

Figura 5. Cambios históricos en las operaciones de pesca de las lanchas pargueras. a) Expansión del área de los caladeros de pesca donde operan las lanchas pargueras, entre 1970 y 2018 y b) Distancia promedio recorrida hasta los caladeros, desde 1970 hasta 2018; las barras de error señalan valores mínimos y máximos.

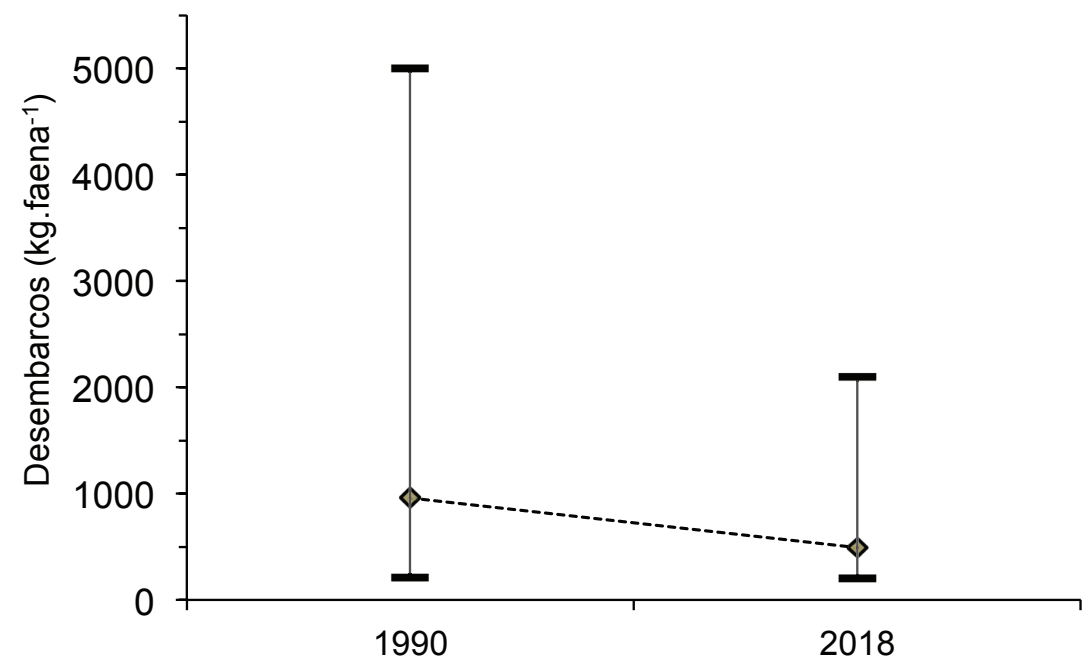

Figura 6. Variación de los desembarcos (kg/faena) obtenida a partir de las declaraciones de los pescadores para 1990 y 2018. La información registra el promedio y las barras de error señalan valores mínimos y máximos. 
La captura por unidad de esfuerzo (CPUE) es uno de los indicadores que puede reflejar la disminución en la abundancia de los recursos explotados. Según la percepción de los pescadores, en casi tres décadas, el promedio de los desembarcos ha disminuido de 957 a 492kg (Figura 6), aspecto que se había evidenciado en las CPUE comparadas por Campo (1996) y Arévalo et al. (2004). En el caso del comportamiento intra-anual de las capturas, los pescadores señalaron que el periodo abril-mayo y septiembre-octubre constituyen los picos para pargo, mero y medregal. Arévalo et al. (2004) describieron, mediante muestreos a bordo, que en la temporada húmeda mayor (septiembre a noviembre), ocurren las mayores capturas con palangres y en la época menor (mayo a junio), las mayores capturas con ballestilla. Esto muestra una alta coincidencia con lo expresado por los pescadores en el presente estudio.

Un aspecto importante que se derivó de las encuestas del CET son las posibles causas de la disminución en las abundancias de los recursos pesqueros aprovechados por la flota parguera. La mayoría (80\%) estuvo asociada a la llegada de la pesca industrial de arrastre de camarón. Los pescadores entrevistados consideraron que los denominados barcos "vikingos" (refiriéndose a los camaroneros), destruyen muchos hábitats, provocando la disminución local de abundancias en estos sitios. Por otro lado, según su apreciación, la disminución de sardinas, como fuente de alimento de los peces más grandes es otra causa importante de la disminución en la abundancia de los recursos objetivo de la flota parguera. Esto último, se contradice con lo reportado por Manjarrés-Martínez \& Cuello (2019), quienes destacan que la distribución espacial del machuelo Opisthonema oglinum tiene una amplia zona de distribución a la que no accede ninguna pesquería orientada, específicamente, a su explotación.

La percepción de los pescadores es que entre 1980 y 2000, el tamaño (longitud total) de los recursos pesqueros, por ellos capturados, ha venido experimentado una disminución. El 80\% de los pescadores entrevistados señalaron que las especies mayormente afectadas resultan ser el pargo ojo amarillo (Lutjanus vivanus) y rojo o legítimo (L. purpureus), así como el mero (Mycteoperca bonaci).

Los resultados evidencian el colapso de esta pesquería artesanal avanzada, cuyos problemas obedecen a la disminución de la abundancia de los recursos, además de los frecuentes problemas que sufren las embarcaciones. El 75\% de los entrevistados coincidió en que las fallas experimentadas por los motores son la principal causa de inactividad de la flota que, muchas veces $(62,5 \%)$, se combina con la falta de liquidez para cubrir los gastos, aunado a problemas de documentos $(25 \%)$ y falta de repuestos $(12,5 \%)$.

Desde su origen, la pesquería artesanal avanzada de lanchas pargueras, que ha operado desde Taganga por casi 50 años, vivió distintas etapas, siempre a la saga de las embarcaciones "pargomeras" venezolanas que, en la década de los 90, alcanzara su apogeo. En la actualidad, el colapso de esta pesquería está asociado a menor abundancia o disponibilidad de los recursos objetivo, aunado a factores económicos, relacionados entre sí: elevados costos de operación, baja rentabilidad, falta de capital, deterioro de cascos y mo- tores, problemas con la documentación (asociados, principalmente, a la expedición de la patente de pesca) de las embarcaciones y falta de repuestos. Esta flota requiere de urgentes medidas de manejo en torno a la extracción de los recursos pargos y meros, pero, principalmente, demanda de una inyección de capital y una acertada gestión empresarial, que mejore los ingresos, reduzca gastos y reoriente sus operaciones hacia una pesquería multipropósito (líneas, nasas y redes), más eficiente y rentable.

Agradecimiento: Los autores agradecen a aquellos patrones de pesca y proeles de las lanchas pargueras de Taganga, que contribuyeron con información valiosa para la realización de esta investigación. Conflicto de intereses: El manuscrito fue preparado y revisado con la participación de todos los autores, quienes declaramos que no existe ningún conflicto de intereses que ponga en riesgo la validez de los resultados presentados. Financiación: Convocatoria interna para la financiación de trabajos de grado de la Vicerrectoría de Investigación de la Universidad del Magdalena.

\section{REFERENCIAS}

1. ARÉVALO, J.; MELO, G.; MARTÍNEZ, L. 2004. Inventario y caracterización general de la flota de lanchas "pargueras" de Taganga, Mar Caribe de Colombia. En: Manjarrés, L. (Ed). Pesquerías demersales del área norte del Mar Caribe de Colombia y parámetros biológico-pesqueros y poblacionales del recurso pargo. Universidad del Magdalena, Santa Marta. p.93-94.

2. BABCOCK, E.; PIKITCH, E.K.; MCALLISTER, M.K.; APOSTOLAKI, P.; SANTORA, C. 2005. A perspective on the use of spatialized indicators for ecosystem-based fishery management through spatial zoning. ICES J. Marine Sci. 62:469-476.

https://doi.org/10.1016/j.icesjms.2005.01.010

3. BARROS, M.; MANJARRÉS, L. 2004. Recursos pesqueros explotados por las pesquerías artesanales marítimas del sector Taganga-La Jorará (Dpto. del Magdalena), con énfasis en peces demersales. En: Manjarrés, L. (Ed). Pesquerías demersales del área norte del Mar Caribe de Colombia y parámetros biológico-pesqueros y poblacionales del recurso pargo. Universidad del Magdalena, Santa Marta. p.93-94.

4. CAMPO, W. 1996. Evaluación del uso de posicionadores por satélite y videosondas en las pesquerías de demersales desarrollada en las "lanchas pargueras" de Taganga. Informe Técnico. Instituto Nacional de Pesca y Acuicultura INPA. Santa Marta. p.32.

5. DE LA HOZ-M., J.; MANJARRÉS-MARTÍNEZ, L.; CUELLO, F. 2015. Estadísticas de desembarco, esfuerzo, valor monetario y rentas de la pesquería artesanal de Taganga período abril-diciembre de 2015. Autoridad Nacional de Acuicultura y Pesca, Bogotá. 32p. 
6. FAO. 2006. Aumento de la contribución de la pesca en pequeña escala a la mitigación de la pobreza y a la seguridad alimentaria. Roma. p.89.

7. FAO. 2018. El estado mundial de la pesca y la acuicultura. 2018. Cumplir los objetivos de desarrollo sostenible. Roma. 233p.

8. GAITÁN-ESPITIA, J. 2008. Estructura de la comunidad del Phylum Echinodermata en aguas someras de la bahía de Taganga, Caribe colombiano. Rev. U.D.C.A Act. \& Div. Cient. 11(1):85-93. https://doi.org/10.31910/rudca.v11.n1.2008.605

9. GÓMEZ-CANCHONG, P.; MANJARRÉS, L.; DUARTE, L.; ALTAMAR, J. 2004. Atlas pesquero del área norte del mar Caribe de Colombia. Universidad del Magdalena. Santa Marta. 230p.

10. GRÜSS, A.; PERRYMAN, H.A.; BABCOCK, E.A.; SAGARESE, S.R.; THORSON, J.T.; AINSWORTH, C.H.; ANDERSON, E.J. 2018. Monitoring programs of the US Gulf of Mexico: inventory, development and use of a large monitoring database to map fish and invertebrate spatial distributions. Reviews in Fish Biology and Fisheries, 28:667-691.

https://doi.org/10.1007/s11160-018-9525-2

11. HALL, G.B.; CLOSE, C.H. 2007. Local knowledge assessment for a small scale fishery, using geographic information systems. Fisheries Research. 83:11-22.

https://doi.org/10.1016/j.fishres.2006.08.015

12. LADDER Research Team. 2001. Methods manual for fieldwork: livelihoods and diversifications explored by research. LADDER Working Paper No. 2. The Overseas Development Group (ODG). 48p.

13. LIMA, M.S.P.; OLIVEIRA, J.E.L.; DE NÓBREGA, M.F.; LOPES, P.F.M. 2017. The use of local ecological knowledge as a complementary approach to understand the temporal and spatial patterns of fishery resources distribution. J. Ethnobiol. Ethnomed. 13(1):30. https://doi.org/10.1186/s13002-017-0156-9

14. MANJARRÉS, L. 1993. Detección de caladeros para la pesca de recursos demersales en La Guajira crucero de septiembre-octubre/ 93. Informe técnico Instituto Nacional de Pesca y Acuicultura INPA. Santa Marta. 315p.

15. MANJARRÉS-MARTÍNEZ, L.M.; CUELLO, F. 2019. Una línea de referencia histórica acerca de la distribución espacial del machuelo (Opisthonema oglinum) en el área de aflora-miento del Mar Caribe de Colombia. Intropica. 14(2):148-159. https://doi.org/10.21676/23897864.3390
16. RAMIRES, M.; CLAUZET, M.; BARRELLA, W.; ROTUNDO, M.M.; SILVANO, R.A.; BEGOSSI, A. 2015. Fishers' knowledge about fish trophic interactions in the southeastern Brazilian coast. J. Ethnobiol. Ethnomed. 11(1):19. https://doi.org/10.1186/s13002-015-0012-8

17. SÁNCHEZ-JIMÉNEZ, A.; FUJITANI, M.; MACMILLAN, D.; SCHLÜTER, A.; WOLFF, M. 2019. Connecting a trophic model and local ecological knowledge to improve fisheries management: The case of Gulf of Nicoya, Costa Rica. Front. Mar. Sci. 6:126. https://doi.org/10.3389/fmars.2019.00126

18. SAAVEDRA-DÍAZ, L.M.; POMEROY, R.; ROSENBERG, A.A. 2016. Managing small-scale fisheries in Colombia. Maritime Studies 15(1):6.

19. SARMIENTO, R.; VÁSQUEZ, E.; BRUNO, M.; FRANCO-HERRERA, A. 2013. Intra-annual variation of climate regime on upwelling zones in the Southeast Caribbean Sea. Rev. Biología Marina y Oceanografía. 48(3):471-485.

http://dx.doi.org/10.4067/S0718-19572013000300006

20. SUÁREZ DE VIVERO, J. 2002. La gobernanza de la pesca en el contexto de la gobernanza marina "conceptos y principales líneas de actuación a escala internacional". JAINA EN LÍNEA. 12:12-16.

21. TEAM Q.D. 2017. QGIS Geographic Information System. Open Source Geospatial Foundation. Disponible desde Internet en: https://qgis.org/es/site/about/index.html\# (con acceso el $18 / 11 / 2018)$.

22. TEH, L.; SUMAILA, U. 2013. Contribution of marine fisheries to worldwide employment. Fish and Fisheries. 14(1):77-88. https://doi.org/10.1111/j.1467-2979.2011.00450.x

23. TEIXEIRA, J.; MARTINS, A.; PINHEIRO, H.; SECCHIN, N.; MOURA, R.; BASTOS, A. 2013. Traditional ecological knowledge and the mapping of benthic marine habitats. J. Environmental Management. 115:241-250. https://doi.org/10.1016/j.jenvman.2012.11.020

24. VILORIA. 1993. Informe técnico Instituto Nacional de Pesca y Acuicultura INPA. Santa Marta. 315p.

25. ZÚÑIGA, H.; ALTAMAR, J.; FERNÁNDEZ, A. 2013. Mejoramiento de la eficiencia en embarcaciones "pargueras" artesanales del caribe colombiano a través de la mecanización de las operaciones de pesca. Rev. U.D.C.A Act. \& Div. Cient. 16(2):469-478.

http://doi.org/10.31910/rudca.v16.n2.2013.921 